\title{
Théologiques
}

\section{La ville : symbolique urbaine et foi chrétienne}

\section{Jean-Guy Nadeau}

Volume 3, numéro 1, mars 1995

Symbolique urbaine et foi chrétienne

URI : https://id.erudit.org/iderudit/602411ar

DOI : https://doi.org/10.7202/602411ar

Aller au sommaire du numéro

Éditeur(s)

Faculté de théologie de l'Université de Montréal

ISSN

1188-7109 (imprimé)

1492-1413 (numérique)

Découvrir la revue

Citer ce document

Nadeau, J.-G. (1995). La ville : symbolique urbaine et foi chrétienne.

Théologiques, 3(1), 5-11. https://doi.org/10.7202/602411ar d'utilisation que vous pouvez consulter en ligne.

https://apropos.erudit.org/fr/usagers/politique-dutilisation/ 
LIMINAIRE

\title{
La ville : symbolique urbaine et foi chrétienne
}

\author{
Jean-Guy NADEAU \\ Faculté de théologie \\ Université de Montréal
}

Les hommes produisent leur ville comme la ville produit ses hommes... Si l'homme est à l'image de la ville, la ville est tout autant à l'image de l'homme..., l'homme qui y naît, qui en naît - et qui en est... La ville n'est pas seulement expressive, elle est naturante... Les hommes... sont, dans leurs gestes et dans leurs démarches, ce que la ville attend d'eux... La ville inspire leurs comportements. Et même leurs rêves; car elle semble aussi gouverner l'imagination humaine; ou plutôt elle secrète elle-même l'imaginaire dont elle s'auréole et par lequel elle signifie. ( M. Dufrenne ${ }^{1}$ )

Cette réflexion toujours actuelle de Mikel Dufrenne exprime bien le projet de ce numéro de Théologiques. L'effet de la ville sur les humains, et donc sur leur expérience de foi, semble aller de soi. Mais on peut se demander dans quelle mesure la réflexion théologique en a intégré les implications. Épitomé d'une modernité qui, elle, constitue toujours un objet de débats théologiques, la ville contemporaine, édifiée par les humains, a radicalement modifié la médiation de l'humain avec le monde et avec lui-même. Et elle continue de le faire quotidiennement. La configuration urbaine secrète un imaginaire particulier, marqué par le quadrillage des rues, la latéralité des boulevards, la verticalité froide et souvent écrasante des gratte-ciel, la puissance de l'automobile, la vitesse du métro, l'affichage publicitaire, le vibrato des néons animant le verre et le béton, les lieux artistiques et culturels nombreux, sans parler des richesses issues de la diversité ethnique urbaine. Partout en ville, l'humain affiche un génie, un pouvoir créateur qui m'émerveille tout autant que celui que manifeste la nature. Il

1 Préface à Pierre SANSOT, Poétique de la ville. Paris, Klinsiek, 1971. 
m'arrive de croire qu'il serait temps que le langage de la foi arrive en ville, quoiqu'il en soit des fautes de l'homo urbanus, Babel n'avait-elle pas de valeur ${ }^{2}$ ?

Mais cette urbanité pose aux citoyens de quelque pays du monde que ce soit des défis toujours plus grands liés à la gestion de grands groupes humains, dont ceux de la coexistence de générations, d'ethnies, de religions, d'éthiques ou de projets divers et souvent en confrontation. Nombre de nos contemporains s'interrogent sur leur ville : la mère de tous les progrès serait-elle devenue la mère de tous les maux? Babylone aurait-elle eu raison de Jérusalem, selon les figures bibliques qu'évoque Norbert Greinacher? La ville contemporaine est-elle déshumanisante, comme on le dit si souvent, opposant sa dynamique sociétaire à une dynamique communautaire dite naturelle (les Gesellschaft et Gemeinschaft que resitue Benoît Bégin ), ou est-elle un lieu original de libération et de réalisation du sujet, voire son lieu d'émergence?

Plusieurs insistent sur les menaces de la ville, décrient l'hégémonie du poulpe urbain qui, étendant partout ses tentacules, a relégué ici la campagne au rang d'un mythe nostalgique ou d'un lieu de retraite tout aussi mythique, alors qu'ailleurs c'est la ville qui fait figure de mythe pour des populations rurales sans cesse appauvries. L'art met largement en scène la déroute du sujet en ville, et la socio-psychologie la difficulté d'y être humain. Les citoyennes et citoyens des grandes villes contemporaines s'inquiètent de la violence et de la pauvreté qui marquent leur milieu et que les média leur transmettent à répétition en les exacerbant. Enquêtes, mass média, littérature rendent amplement compte de cette violence et de cette inquiétude qui marquent le roman américain, le roman policier, la science-fiction ( surtout le cyberpunk), la danse et le théâtre contemporains. D'autres croient encore aux possibilités de la ville, y demeurent, y immigrent, $y$ travaillent ou y luttent, parfois portés par leur foi, pour en rendre les conditions de vie plus humaines, plus viables.

De villes organisées autour du sacré, nous sommes passés à des villes où le sacré semble absent, ou revêtir de nouvelles figures, "profanes " : temple des affaires, idoles du sport ou du rock, ziggourats des multinationales, etc. C'est le plus souvent en fonction des affaires ou du " marché du travail " que s'établissent les rapports urbains actuels. C'est que l'imaginaire urbain n'est pas qu'architectural ou spatial. Il s'établit aussi en fonction de parti-pris économiques, politiques, éthiques que certains articles de ce numéro, je pense particulièrement à celui de Benoît Bégin, mettent bien en lumière. Or ce marché des affaires, comme notre civilisation urbaine, est autoréférentiel, sans transcendance ni archéologique ni téléologique ( si ce n'est le télos du plus ). Le vouloir

2 François MARTY, La bénédicion de Babel. Paris, Cerf, 1990. 
humain et ses capacités - peut-être serait-il plus juste de parler d'appétit, de convoitise, voire de boulimie ( " faim excessive accompagnant certains troubles physiques ou mentaux ", dixit le Petit Robert ) - y sont la mesure. On ne peut douter de l'impact de cette situation sur la relation de l'être humain avec luimême, sur sa capacité de relation avec le transcendant, sur ses rapports à la vérité, etc. Épitomé du désenchantement du monde, la ville contemporaine s'oppose-t-elle in se au religieux? Son imaginaire n'est-il que d'immanence ou ouvre-t-il, fut-ce à revers, sur d'autres horizons?

Chose certaine pour plusieurs, la ville contemporaine rend caduques les expressions religieuses, particulièrement celles de la foi chrétienne qui paraissent relever de civilisations nomades ou agricoles, impériales ou féodales révolues. Pour d'autres, c'est, au-delà du langage, la possibilité même de la foi qui s'y trouve mise en cause. Plus précisément, la foi chrétienne est-elle toujours possible en notre contexte urbain industrialisé et technologisé, ou relève-t-elle de visions du monde dont les supports se seraient écroulés avec le développement de la ville? Par ailleurs, même si on ne saurait nier la contextualité de l'expérience de foi, il semble au croyant qu'on ne saurait la réduire à des effets de contexte. De plus, de nouvelles religions naissent et proliferent en ville, dont certaines semblent portées par une nostalgie religieuse, d'autres par un désir justement de s'opposer à la froideur, à l'anonymat, à l'enfermement urbain autoréférentiel. La théologie, plus que la pastorale, a certes pris note de ce mouvement de désenchantement, par exemple à travers la thématique de la sécularisation ou encore à travers la distinction, parfois l'opposition, entre la religion et la foi. Mais il semble qu'elle n'en a pas encore envisagé toutes les conséquences, les défis, les avenues. Or, la ville détermine sans doute aussi, mais souvent à notre insu, les recherches théologiques quant à leurs objets, à leurs méthodes, à leurs perspectives. L'absence d'une telle détermination serait certes dramatique, mais avons-nous bien pris la mesure de ses effets, par exemple sur l'herméneutique et l'épistémè contemporaines?

Voilà, brièvement, quelques-unes des questions à la source de ce numéro de Théologiques qui vise à apporter sa contribution à une question malheureusement laissée en friche il y a plusieurs années. On aura compris qu'il ne s'agit pas de faire le procès de la ville contemporaine mais d'explorer quelques aspects de celle-ci et de réfléchir à leur articulation avec le monde de la foi chrétienne.

À plusieurs plans, la ville apparaît comme le lieu de l'autre, le lieu où chacun est manifestement un autre, pour peu qu'il emprunte les transports en commun ou déambule sur la rue. Le "monde de la rue ", par exemple, inquiète 
et fascine à la fois ( fascinans et tremendum ) plusieurs de nos contemporains. Sa population de sans-abris et de " jeunes de la rue "fait problème partout à travers le monde. $\grave{A}$ un point tel que, dans certaines villes, des escouades armées se vouent à leur extermination. Monde dur, la rue est un lieu fascinant, excitant en même temps qu'inquiétant, parfois scandalisant (dans l'apathie du standerby, par exemple). Lieu de passage, de quête, de crime, d'initiation et d'identification, la rue est aussi lieu de rencontre (de "divine rencontre", affirment des écrivains ), de vie, de libération, de festivals, de manifestation, de contrôle et d'intervention ( même pastorale). La rue apparaît comme la vitrine de la ville, le lieu où celle-ci expose ses passions, ses scories, son imaginaire. Lieu de l'image, la rue donne aussi à voir ce que la société ne voudrait pas voir mais qui fascine par ses marques d'altérité, telle la prostituée que Pierre Sansot désignait comme un bouton de chair à travers le béton des villes. Comme quoi la modernité urbaine n'a pas tout bulldozé. Mais combien souhaiteraient qu'elle le fasse, voulant s'approprier toujours davantage leur ville! C'est que l'espace urbain apparaît comme un bien, à s'approprier ou à sauvegarder, en refoulant par exemple les prostituées vers d'autres quartiers que le sien. Comme en province on refoule les $\mathrm{BPC}$ vers d'autres régions que la sienne.

La contribution de Daniel Welzer-Lang et Laurette Wittner et celle de Myriame El Yamani décrivent ce qui se passe lorsque " ma ville " s'avère être aussi la ville de l'autre. Alors, l'espace, l'espace de rencontre, devient enjeu de lutte, au mieux de négociation. Et réapparaissent, sur un horizon différent, les "chicanes de clochers" d'antan. Si la ville marque notre imaginaire, il est clair que celui-ci, et d'abord au plan de la propriété ou d'un certain droit à l'espace, marque l'aménagement de la ville. Du rapport de forces alors en jeu, il s'ensuit que certains se trouvent étrangers dans leur propre ville, dépossédés de leur ville, une ville qui n'a peut-être en fait jamais été la leur même s'ils y habitent. L'architecture de certains quartiers, le tracé des rues, le vêtement, la couleur de la peau ou la parlure déterminent non seulement la vie des habitants de la ville mais aussi leurs relations. L'aménagement, le contrôle de l'espace urbain indique bien que la ville a différentes classes de citoyens et citoyennes. Rien de nouveau, comme en témoigne l'histoire, dans cette tension entre la ville centripète, espace ouvert à la circulation de tous, et le refoulement de groupes entiers hors du centre ou de certains quartiers, la tension entre le contrôle et son absence selon le groupe d'appartenance manifeste des citoyens.

La multi-ethnicité et le pluralisme religieux, qui tendent à constituer un trait dominant des grandes villes du monde, sont source de nouveaux défis. Or, on ne fait que commencer à prendre conscience de leurs effets sur le paysage et l'imaginaire urbains. Amenant au citadin l'universalité chez soi, cette multiethnicité urbaine semble multiplier les occasions de racisme et de violence, en même temps que de solidarité. Si la violence, certains diront la provocation, 
semble l'emporter, on peut se demander si cette situation ne pourrait devenir lieu d'apprentissage de l'altérité et d'un nouveau vivre ensemble. Ce qui peut apparaître comme un vœu pieux s'avère en fait relever de la nécessité. Certes, tous les citoyens n'y sont pas encore confrontés. Soit parce que leur ville n'a pas la taille voulue ou reste à l'écart des grandes voies de migration internationales, soit parce qu'ils ne quittent pas ou peu un quartier qui " a la chance ", diront ses habitants, d'échapper à ces mouvements de population. Mais ce n'est un secret pour personne que le régime pédagogique et le milieu de vie scolaire, par exemple, sont remis en question par ce bouillonnement démographique où se mêlent des populations et des cultures étrangères voire hostiles les unes aux autres. Même les paroisses des grandes villes n'y échappent pas, qui ont vu leur population se modifier radicalement au cours des dernières années avec la croissance notable de groupes ethniques avec lesquels ces paroisses n'arrivent plus à entrer en contact. Tout se passe alors comme si le territoire paroissial se rétrécissait de fait, confronté non seulement à d'autres codes culturels mais aussi, et de plus en plus, à d'autres codes religieux. Voilà que se pose chez nous la question des rapports entre les religions, alors que nous avons à peine relevé les défis liés aux rapports entre les confessionnalités chrétiennes.

Si la rue apparaît parfois comme menaçante, les édifices religieux qui la jalonnent apparaissent à plusieurs comme des havres de paix, de culture. Par exemple, bien qu'on ne saurait dissocier radicalement le culte de la culture, certaines églises à travers le monde sont beaucoup plus fréquentées pour des activités culturelles que pour des activités cultuelles. Cette situation témoigne sans doute d'un écart entre le culte chrétien et la culture actuelle, mais peut-être aussi de l'aptitude de ces lieux religieux à rester des lieux d'ouverture sur l'audelà dans une civilisation urbaine auto-suffisante. Les sanctuaires urbains apparaissent comme des oasis de transcendance perçant l'immanence de la cité. Une de nos collègues, professeure en criminologie, nous avait suggéré un article sur la nouvelle utilisation, voire la nouvelle signification des églises en ville. Elle n'a malheureusement pas pu donner elle-même suite à sa suggestion. André Lamoureux, responsable d'une de ces églises ouvertes sur la culture, a accepté de nous communiquer son expérience, certes particulière, mais qui a l'intérêt de répondre aux questions que d'aucuns, oubliant l'histoire, se posent quant à l'usage " profane " de lieux religieux. On peut même se demander si la vocation culturelle de tels lieux, où se rencontrent l'esthétique religieuse et la création contemporaine, ne sauve pas une partie de leur vocation religieuse en les désignant comme un lieu où, à la jonction d'atmosphères profanes et sacrées, s'ouvre une imagination dont on a oublié les possibilités religieuses.

Si le christianisme semble avoir peu de rapports avec l'esthétique contemporaine, on peut aussi s'interroger sur le rapport que le langage évangélique entretient avec l'urbanité contemporaine. Les villes de l'Empire où s'est déve- 
loppée la foi chrétienne avaient bien peu à voir avec nos cités post-industrielles. D'où notre demande à André Myre de présenter en fonction de notre thème l'ouvrage de Ched Myers, Binding the Strong Man. A Political Reading of Mark's Story of Jesus, de façon à resituer les rapports entre l'imagerie rurale de l'Évangile et le monde urbain contemporain. L'Évangile, en effet, et c'est là un de ses effets poétiques majeurs au-delà des images dont on ne saurait toutefois ignorer le poids, appelle à une solidarité qui semble d'autant actuelle que l'Évangile de Marc dénonce l'économie politique d'une ville centrée sur le temple qui représentait pour Marc le cour de ce qui n'allait pas dans le système social de son temps (Myers, p. 80). Or, nous le disions plus haut, le temple abrite aujourd'hui d'autres divinités et l'Évangile est toujours critique de sa domination politique, que ce temple soit religieux ou financier.

Il n'en reste pas moins que la pastorale relève d'abord d'un monde préurbain, même pour un Michel Foucault qui considère que " la pastorale des âmes est une expérience typiquement urbaine ${ }^{3}$. » D'où notre interrogation sur la performativité "poétique " d'un tel terme dans nos contextes urbains. "Chantez-vous encore la chanson du mouton?", demandait quelqu'un en parlant de l'Agnus Dei. L'image pastorale du troupeau et de son guide semble avoir fait son temps, quoiqu'elle désigne bien la mentalité des sectes contemporaines. N'est-elle qu'anachronisme ou peut-elle toujours s'avérer féconde dans le contexte urbain actuel? Par ailleurs, si la pastorale critique l'individualisme et ses effets sur l'appartenance, les croyances et les pratiques religieuses, on a moins exploré l'effet de la pastorale elle-même sur l'individuation des citoyens, comme l'a suggéré Foucault en y reliant l'origine de la police ( au sens large, d'abord ) et de l'état providence 4 .

Comme le signale André Charron, l'Église urbaine est confrontée à un nouveau marché individualiste du religieux, concomitant au marché du travail et de la consommation. Un marché sur lequel elle a en quelque sorte perdu son monopole, où elle n'est plus le seul producteur de sens religieux. Comme toute situation de non monopole, comme toute situation pluraliste, celle que nous connaissons peut tout aussi bien mener à la relativisation de toute appartenance qu'à l'engagement personnel dans un ou l'autre des lieux de sens religieux qui s'offrent à nos contemporains. Mais on peut aussi se demander à la lecture de la

3 Michel FoucAULT, « Omnes et singulatim : vers une critique de la raison politique *, Le Débat 41 (1986) 21.

4 Outre l'article précédent, voir Michel FOUCAULT, * Pourquoi étudier le pouvoir: la question du sujet ", dans H. DREYFus et P. RABINOW, Michel Foucault. Un parcours philosophique (avec un entretien et deux essais de Michel Foucault). Paris, Gallimard, 1984, p. 297-308. 
contribution d'André Charron dans quelle mesure la pastorale chrétienne, alors même qu'elle la critique, s'est modelée sur la civilisation urbaine et son marché actuel : religion à la carte, consommation sacramentelle, création de groupes religieux, etc. Le propos de vouloir assumer la ville recèle de multiples possibilités d'organisation, d'appartenance, de culte et d'engagement. Il interpelle chrétiens et chrétiennes, comme la communauté à laquelle ils veulent bien apparrenir, à travailler à l'avènement de la nouvelle Jérusalem, figure urbaine du Royaume de Dieu dont on rêve qu'il marque enfin nos rapports à autrui.

Ce sont finalement ces rapports à autrui qui s'avèrent l'enjeu majeur de ce numéro que nous avions pourtant orienté sur le rapport au religieux. On ne s'en étonnera peut-être pas; d'une part parce que nous sommes en ville, d'autre part parce que le rapport au religieux en christianisme mène à une éthique des relations humaines, voire de solidarité humaine. La ville contemporaine pose peutêtre plus que jamais la question du rapport à l'autre, à l'étranger, au différent qu'elle impose de plus en plus à ses habitants, de même que le rapport à la logique économique qui détermine largement ces relations à l'autre. La symbolique chrétienne a-t-elle suffisamment pris la mesure de la ville, de façon à être signifiante dans cette quête de nouveaux rapports humains qui constitue un défi majeur tant pour la théologie que pour l'intervention et la recherche sociales et pastorales? 